\title{
THE LICK AGN MONITORING PROJECT: VELOCITY-DELAY MAPS FROM THE MAXIMUM-ENTROPY METHOD FOR Arp 151
}

\author{
Misty C. Bentz ${ }^{1,11}$, Keith Horne ${ }^{2}$, Aaron J. Barth ${ }^{1}$, Vardha Nicola Bennert ${ }^{3}$, Gabriela Canalizo ${ }^{4,5}$, \\ Alexei V. Filippenko ${ }^{6}$, Elinor L. Gates ${ }^{7}$, Matthew A. Malkan ${ }^{8}$, Takeo Minezaki ${ }^{9}$, Tommaso Treu $^{3}$, Jong-Hak Woo ${ }^{10}$, \\ AND JONELle L. WALSH ${ }^{1}$ \\ ${ }^{1}$ Department of Physics and Astronomy, 4129 Frederick Reines Hall, University of California, Irvine, CA 92697, USA; mbentz@uci.edu \\ 2 SUPA Physics and Astronomy, University of St Andrews, North Haugh, St Andrews KY16 9SS, UK \\ ${ }^{3}$ Physics Department, University of California, Santa Barbara, CA 93106, USA \\ ${ }^{4}$ Institute of Geophysics and Planetary Physics, University of California, Riverside, CA 92521, USA \\ ${ }^{5}$ Department of Physics and Astronomy, University of California, Riverside, CA 92521, USA \\ ${ }^{6}$ Department of Astronomy, University of California, Berkeley, CA 94720-3411, USA \\ ${ }^{7}$ Lick Observatory, P.O. Box 85, Mount Hamilton, CA 95140, USA \\ ${ }^{8}$ Department of Physics and Astronomy, University of California, Los Angeles, CA 90024, USA \\ ${ }^{9}$ Institute of Astronomy, School of Science, University of Tokyo, 2-21-1 Osawa, Mitaka, Tokyo 181-0015, Japan \\ ${ }^{10}$ Astronomy Program, Department of Physics and Astronomy, Seoul National University, Gwanak-gu, Seoul 151-742, Republic of Korea \\ Received 2010 May 27; accepted 2010 July 5; published 2010 August 11
}

\begin{abstract}
We present velocity-delay maps for optical H I, He I, and He II recombination lines in Arp 151, recovered by fitting a reverberation model to spectrophotometric monitoring data using the maximum-entropy method. H I response is detected over the range 0-15 days, with the response confined within the virial envelope. The Balmer-line maps have similar morphologies but exhibit radial stratification, with progressively longer delays for $\mathrm{H} \gamma$ to $\mathrm{H} \beta$ to $\mathrm{H} \alpha$. The He I and He II response is confined within 1-2 days. There is a deficit of prompt response in the Balmer-line cores but strong prompt response in the red wings. Comparison with simple models identifies two classes that reproduce these features: free-falling gas and a half-illuminated disk with a hot spot at small radius on the receding lune. Symmetrically illuminated models with gas orbiting in an inclined disk or an isotropic distribution of randomly inclined circular orbits can reproduce the virial structure but not the observed asymmetry. Radial outflows are also largely ruled out by the observed asymmetry. A warped-disk geometry provides a physically plausible mechanism for the asymmetric illumination and hot spot features. Simple estimates show that a disk in the broad-line region of Arp 151 could be unstable to warping induced by radiation pressure. Our results demonstrate the potential power of detailed modeling combined with monitoring campaigns at higher cadence to characterize the gas kinematics and physical processes that give rise to the broad emission lines in active galactic nuclei.
\end{abstract}

Key words: galaxies: active - galaxies: individual (Arp 151) - galaxies: nuclei - galaxies: Seyfert

Online-only material: color figures

\section{INTRODUCTION}

Active galactic nuclei (AGNs) have long been known to undergo dramatic and rapid variations in brightness (Matthews \& Sandage 1963; Smith \& Hoffleit 1963). This variability is exploited with the technique of reverberation mapping (Blandford \& McKee 1982; Peterson 1993) to study the broad-line region (BLR) of AGNs, a region of gas only $\sim 0.01 \mathrm{pc}$ in size and presumed to be photoionized by the emission from an accretion disk around a supermassive black hole. Spatially unresolvable even for the most nearby galaxies, the BLR is studied in the time domain through monitoring the variable continuum emission (presumably originating close to the black hole) and its reprocessing in the BLR gas, discernible as variations in the broad emission-line fluxes and profiles.

Under the assumptions that (1) the continuum-emission region is much smaller than the BLR, (2) time delays arise predominantly from light-travel time, and (3) the relationship between the ionizing and observed continuum is smooth, a linearized echo model can be used to express changes in the emission lines $(\Delta L)$ as time-delayed responses to changes in the

\footnotetext{
${ }^{11}$ Hubble Fellow
}

continuum $(\Delta C)$ :

$$
\Delta L(v, t)=\int_{0}^{\infty} \Psi(v, \tau) \Delta C(t-\tau) d \tau .
$$

Here, $\Delta L(v, t)$ is the change in the emission-line flux at line-ofsight velocity $v$ and time $t, \Delta C(t)$ is the change in the continuum flux at time $t$, and $\Psi(v, \tau)$ is the so-called transfer function, or velocity-delay map, giving the distribution of the line response over line-of-sight velocity $v$ and time delay $\tau$ (i.e., the emissionline response to a $\delta$-function continuum event). Information on the BLR geometry, kinematics, and ionization structure is encoded in $\Psi(v, \tau)$, making recovery of velocity-delay maps a key goal of echo-mapping experiments.

Due to the formidable requirements of long-duration monitoring, high temporal sampling, and high-quality and homogeneous data for recovery of velocity-delay maps (Horne et al. 2004), most echo-mapping experiments have restricted the analysis to a simpler problem: measuring the mean delay $\tau$, and hence the mean radius $R \approx c \tau$ of the BLR, through cross-correlation of the line and continuum light curves (e.g., Antonucci \& Cohen 1983; Peterson et al. 1983, 2004; Kaspi et al. 2000). Analyses comparing delays in different parts of the emission-line profiles, such as the red wing versus the blue wing (e.g., Gaskell 1988; Koratkar \& Gaskell 1989; Crenshaw \& Blackwell 1990), have 
sometimes shown evidence of asymmetry, hinting at gross characteristics (inflow versus outflow versus circulation) of the BLR gas flow.

For certain high-quality data sets, delay maps $(\Psi(\tau)=$ $\left.\int \Psi(v, \tau) d v\right)$ have been recovered. These include delay maps for Ly $\alpha$ and C IV, among other lines, derived from the International Ultraviolet Explorer (IUE) monitoring data set for NGC 5548 (Krolik et al. 1991), and the delay maps recovered by Horne et al. (1991) from ground-based optical monitoring of $\mathrm{H} \beta$ in NGC 5548. The delay maps generally agree with the results of cross-correlation analysis, while allowing additional insights into the details of the BLR. Horne et al. (1991) find a lack of $\mathrm{H} \beta$ response at zero time delay in NGC 5548, a signature of anisotropic (inward) $\mathrm{H} \beta$ response, and/or a paucity of BLR gas near the line of sight.

In addition to presenting delay maps for several ultraviolet lines in NGC 4151, Ulrich \& Horne (1996) also present a partially recovered velocity-delay map for C IV, which unfortunately suffered from strong intrinsic absorption in the line core throughout the campaign. Three separate groups attempted to recover velocity-delay maps from an IUE and Hubble Space Telescope monitoring campaign of NGC 5548; however, these studies were again hampered by the quality of the $C_{\text {IV }}$ data used, and the final conclusions ranged from no radial motion (Wanders et al. 1995) to some radial infall (Done \& Krolik 1996) to radial outflow (Chiang \& Murray 1996; Bottorff et al. 1997).

Recent results from the Lick AGN Monitoring Project (LAMP; Bentz et al. 2008, 2009) and from MDM Observatory (Denney et al. 2009, 2010) have shown that recovery of velocity-delay maps may be within the reach of current AGN monitoring programs due to their monitoring baselines of several months, daily sampling, and homogeneous data with high signal-to-noise ratios $(\mathrm{S} / \mathrm{N})$. In this Letter, we present velocitydelay maps recovered with maximum-entropy techniques for six optical $\mathrm{H}$ and $\mathrm{He}$ recombination lines in the LAMP spectra of Arp 151 (Mrk 40): $\mathrm{H} \alpha, \mathrm{H} \beta, \mathrm{H} \gamma$, He I $\lambda 5876$, He I $\lambda 4471$, and He II $\lambda 4686$. We also suggest plausible models for the geometry and kinematics of the BLR in Arp 151 that could account for the observed velocity-delay structure.

\section{OBSERVATIONS}

Details of the photometric light curves are presented by Walsh et al. (2009). In brief, broad-band Johnson $B$ and $V$ images of Arp 151 were obtained at the $0.8 \mathrm{~m}$ Tenagra II telescope in southern Arizona between 2008 February 27 and May 16 (UT here and throughout). Standard reduction techniques were employed, and photometric light curves were measured with differential photometry relative to field stars. Absolute flux calibrations were determined using Landolt (1992) standardstar fields.

Spectroscopic monitoring of Arp 151 was carried out at the Lick Observatory $3 \mathrm{~m}$ Shane telescope with the Kast dual spectrograph between 2008 March 25 and May 21 (see Bentz et al. 2009 for details). The Kast red-side CCD with the 600 lines $\mathrm{mm}^{-1}$ grating allowed spectral coverage over the range 4300-7100 A. Spectra were obtained at a fixed position angle of $90^{\circ}$ through a $4^{\prime \prime}$ wide slit, with a typical $\mathrm{S} / \mathrm{N} \approx 100$ per pixel at rest frame $5100 \AA$. Flux calibrations were determined from nightly spectra of standard stars.

Small time-variable and wavelength-dependent corrections were made to the spectra to account for three types of systematic error: (1) wavelength shifts due to flexure and atmospheric differential refraction, (2) spectral blurring due to atmospheric seeing and instrumental resolution, and (3) photometric errors due to slit losses and atmospheric transmission (see M. C. Bentz et al. 2010, in preparation, for a detailed description of the methodology). After correcting for these errors, the continuum-subtracted line-profile variations were subjected to echo-mapping analysis with MEMECHO.

\section{MEMECHO LIGHT-CURVE MODELING}

MEMECHO (see Horne et al. 1991; Horne 1994 for details) uses a maximum-entropy technique to recover $\Psi(v, \tau)$ by fitting the linearized echo model of Equation (1) to the observed continuum light curve and responding emission-line velocity profiles. MEMECHO finds the "simplest" positive image $p_{i}$ that fits the data. The image $p_{i}$ includes an evenly sampled driving continuum light curve $C(t)$, a background spectrum $L_{0}(\lambda)$ representing non-variable line emission, and the velocitydelay map $\Psi(v, \tau)$. The quality of the fit to the $N$ data points is measured by $\chi^{2}$ summed over measurements of the driving continuum light curve and the reverberating emissionline spectrum. Simplicity is measured by the entropy $S=$ $\sum_{i} p_{i}-q_{i}-p_{i} \ln \left(p_{i} / q_{i}\right)$ relative to a "default image" $q_{i}$. Since $\partial S / \partial p_{i}=-\sum_{i} \ln \left(p_{i} / q_{i}\right)$, maximum entropy at $S=0$ occurs when $p_{i}=q_{i}$. Our default image $q_{i}$ slightly blurs the image $p_{i}$ (for example, $q_{i}=\sqrt{p_{i-1} p_{i+1}}$ for a one-dimensional image), and thus $S$ increases toward 0 for smooth positive images. With suitable data sets, MEMECHO delivers a well-defined map maximizing $S$ for a specified $\chi^{2} / N$. The resolution of the map and the quality of the fit improve as $\chi^{2} / N$ is reduced. When $\chi^{2}$ is set too low, the fit attempts to follow noise in the data, resulting in noisy maps. The maps we show are for $\chi^{2} / N=1.1$, giving the highest resolution maps and the best fits we could achieve while avoiding the introduction of spurious structure, typically large excursions in the gaps between measurements of $C(t)$.

We chose the $B$-band photometric light curve as the driving continuum light curve for the MEMECHO analysis. The variations in $B$ are stronger than in $V$ with no evident time delay between the two (Walsh et al. 2009), and the photometric light curves are more accurately calibrated than the spectroscopic continuum light curve, which suffers from aperture effects. Furthermore, the contribution of the broad emission lines to the photometric variability is negligible (see Walsh et al. for a complete discussion). The $V$-band light curve and the integrated $\mathrm{H} \alpha, \mathrm{H} \beta$, and $H \gamma$ light curves were then modeled as echoes of $B$, as were the emission-line light curves at each wavelength. Thus, we fit delay distributions simultaneously to a total of 1373 individual light curves-one per spectral pixel (each with $\Delta \lambda=2 \AA$ ), plus the $V$-band and integrated Balmer-line light curves. We enforce causality by requiring non-negative delays, $\tau_{\min }=0$ days. We set $\tau_{\max }=20$ days, which is $\sim 1 / 3$ of the spectroscopic monitoring baseline, and we use a uniform spacing of $\Delta t=0.5$ days in the delay maps and continuum light curve. The linearized echo model gives a tangent-line approximation to what may in principle be nonlinear responses to changes $\Delta C(t)=C(t)-C_{0}$ away from a reference continuum level $C_{0}$. For $C_{0}$, we adopt the mean of the observed $B$-band fluxes.

In Figure 1, we show selected light curves of Arp 151 along with their MEMECHO fits and recovered delay maps. The selected wavelengths sample the cores of the emission lines plus the Balmer-line wings. The $B$-band (driving) light curve is shown in the bottom panel. The value of $\chi^{2} / N$ is reported for each light curve shown, and is 1.1 when summed over all light curves including those not shown. 


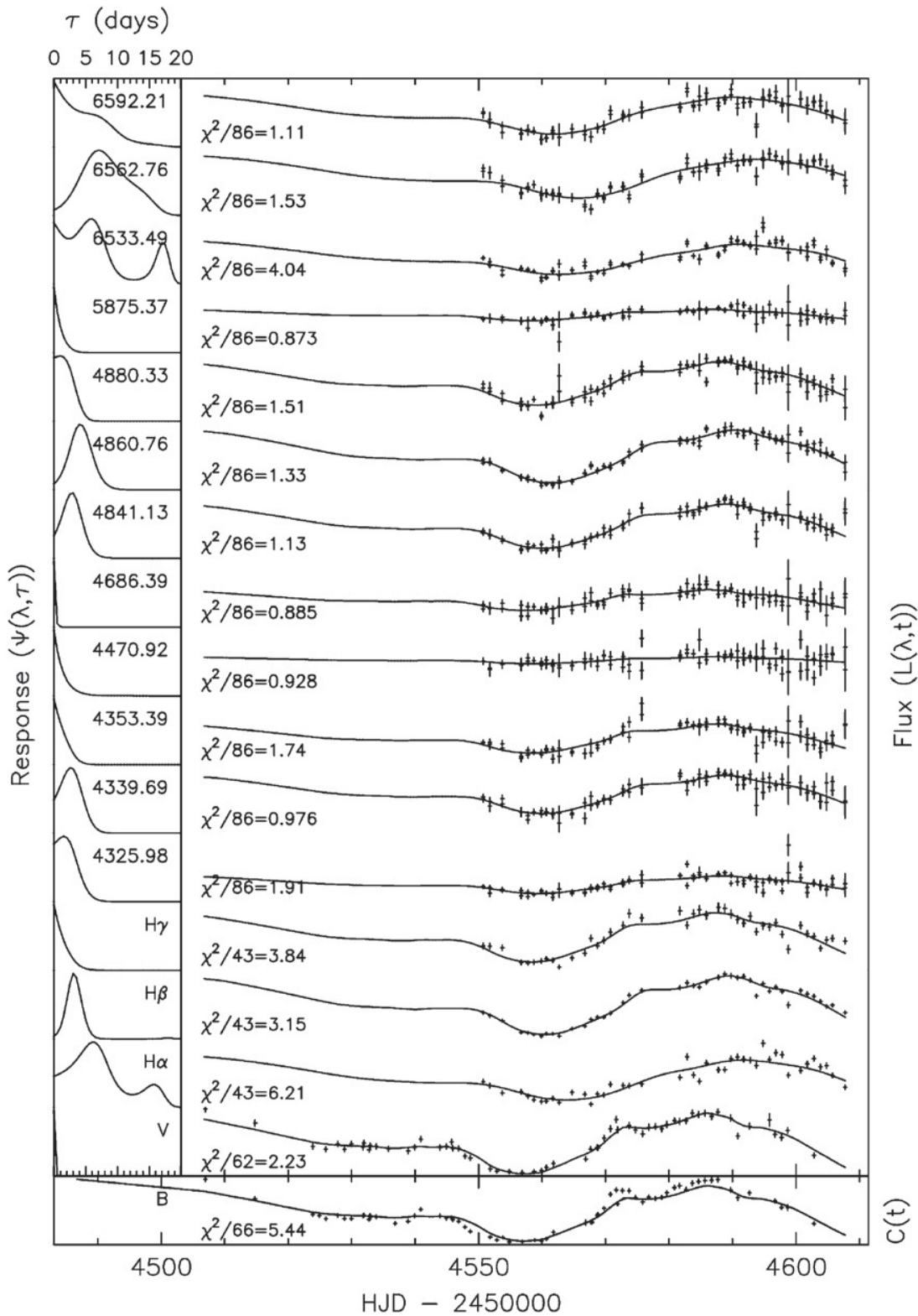

Figure 1. MEMECHO fits to the light curves at selected wavelengths in the spectra of Arp 151 (right panel) and their delay maps (left panel). The rest-frame central wavelength of each pixel is listed with its delay map, and the $\chi^{2} / N$ values are listed with the light curves. We include four supplementary integrated light curves ( $V$ band, $\mathrm{H} \alpha, \mathrm{H} \beta$, and $\mathrm{H} \gamma$ ) as fitting constraints, which are displayed near the bottom. Multiple measurements in the same night are averaged together in the integrated light curves, but are treated separately for the non-integrated light curves.

\section{THE VELOCITY-DELAY MAPS}

The delay maps at each wavelength are assembled into a velocity-delay map shown over the full spectral range in Figure 2 and centered on the six strongest emission lines in Figure 3. To aid in comparing the velocity-delay structure of different emission lines, Figure 4 presents false-color maps with red, green, and blue colors giving the velocity-delay distributions of different emission lines as indicated.

Taken together, the velocity-delay maps exhibit a virial structure with the emission-line response covering a wider range of velocities at smaller delays. This is perhaps best seen in Figures 3 and 4, where the line responses are confined within the "virial envelope" $v^{2}=G M / c \tau$ for $M_{\text {virial }}=1.2 \times 10^{6} M_{\odot}$ (Bentz et al. 2009).

The Balmer-line maps all have the same basic structure, with longer delays in the core and smaller delays in the line wings, and with the red-wing response much stronger than that of the blue wing. The $\mathrm{H} \alpha$ response is detected from 0 days in the red wing to about 15 days in the line core. The other Balmer lines have a similar structure, but with $\mathrm{H} \beta$ extending to about 7 days and $\mathrm{H} \gamma$ to 5 days. The mean delays from these maps are consistent with the mean lag times from the cross-correlation analysis presented by Bentz et al. (2010). A plausible interpretation of this effect is a radial stratification resulting from optical-depth effects within the Balmer series (Korista \& Goad 2004).

The response of the helium lines is consistent with zero time delay, except for some response at small positive delays in the map of He I $\lambda 5876$ and $\mathrm{He}$ I $\lambda$ 4471. As discussed by Bentz et al. (2010), the helium response occurs on shorter timescales than our monitoring sampling and is therefore unresolved.

The H $\alpha$ map shows a "curl" at long time delays on the blue side of the line outside the virial envelope. This weak feature may be an artifact, as it does not always appear in maps made with slightly different fitting parameters. Features that appear 


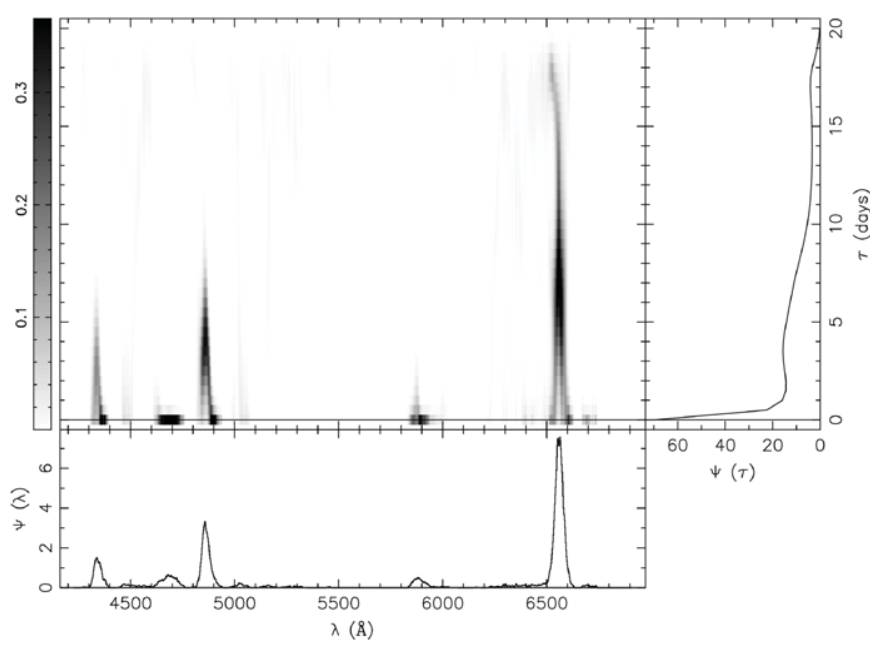

Figure 2. Velocity-delay map $\Psi(\lambda, \tau)$ over the full wavelength range (grayscale), and projections onto the wavelength axis $\Psi(\lambda)=\int \Psi(\lambda, \tau) d \tau$ (bottom) and the time-delay axis $\Psi(\tau)=\int \Psi(\lambda, \tau) d \lambda$ (right).

in all of the Balmer lines, such as the strong emission in the red wings, are more secure.

The maps show a lack of prompt response in the core of all three Balmer lines. The delay map for the integrated $\mathrm{H} \beta$ profile in Figure 1 shows a paucity of response at zero lag, similar to what was found for NGC 5548 (Horne et al. 1991), even though the full velocity-delay map clearly shows gas responding with zero lag in the red wing of $\mathrm{H} \beta$.

\section{DISCUSSION}

We have examined the predicted velocity-delay structure for a variety of simple BLR models to identify classes of models that fail and succeed in qualitatively reproducing the main features of the observed velocity-delay maps. Two successful classes of models are represented by the Free-fall and Disk + Hot spot
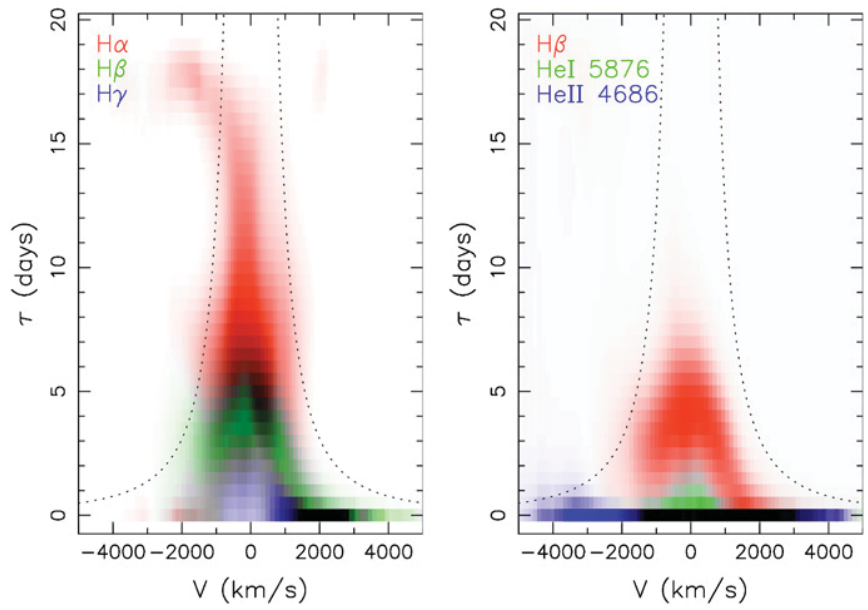

Figure 4. Left: comparison of the velocity-delay maps for $\mathrm{H} \alpha$ (red), $\mathrm{H} \beta$ (green), and $\mathrm{H} \gamma$ (blue). Right: comparison of the velocity-delay maps for $\mathrm{H} \beta$ (red), He I $\lambda 5876$ (green), and He II $\lambda 4686$ (blue). As in Figure 3, the dotted lines in each panel show the virial envelope. The Balmer lines all have the same basic shape, but are graduated in their response ranges. The helium-line response is mostly unresolved in time.

(A color version of this figure is available in the online journal.)

models shown in Figure 5. For all four models in Figure 5, we adopt $M_{\mathrm{BH}}=7 \times 10^{6} M_{\odot}$ (assuming $f=5.5$; Bentz et al. 2009).

A thin spherical shell of radius $R$ infalling with velocity $V$ covers a sloped line on the velocity-delay map, with $\tau=$ $(1-\cos \theta) R / c$ and $v=V \cos \theta$, the delay increasing from $\tau=0$ at $v=+V$ on the near side to $\tau=2 R / c$ at $v=-V$ on the far side of the shell. The Free-fall model in Figure 5 has a spherical distribution of infalling gas with $R_{\text {in }}=0.1$ lt-days and $R_{\text {out }}=4$ lt-days, and $V=\sqrt{G M / R}$, giving small delays on the red side and longer delays with a virial envelope on the blue side of the map. This naturally produces the required asymmetry. The lack of prompt response in the line core can arise by means
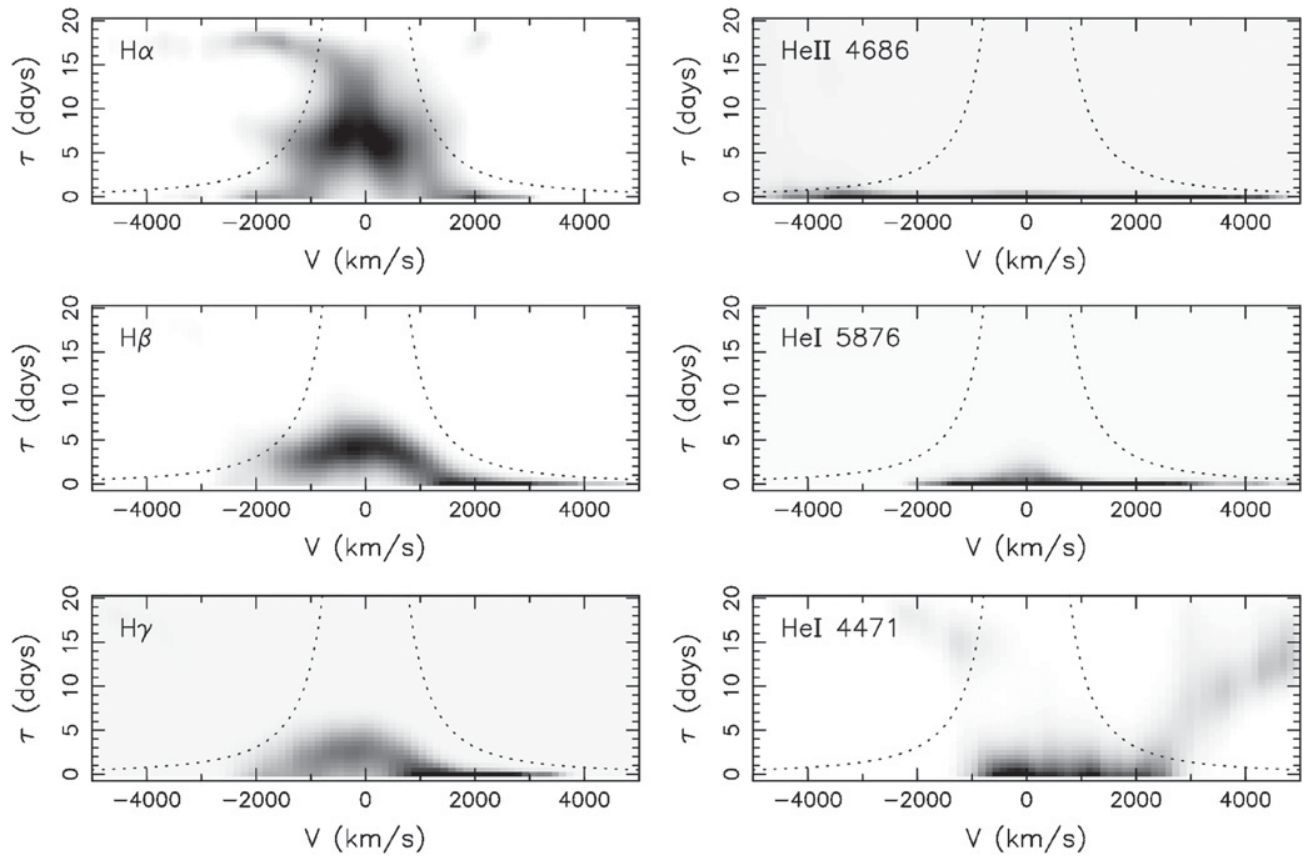

Figure 3. Velocity-delay maps for each of the six optical $\mathrm{H}$ and He recombination lines in the LAMP spectra of Arp 151. The dotted lines in each panel show the "virial envelope" $V^{2} \tau c / G=1.2 \times 10^{6} M_{\odot}$, based on the "virial product" of time lag and line width for $\mathrm{H} \beta$ (Bentz et al. 2009). 

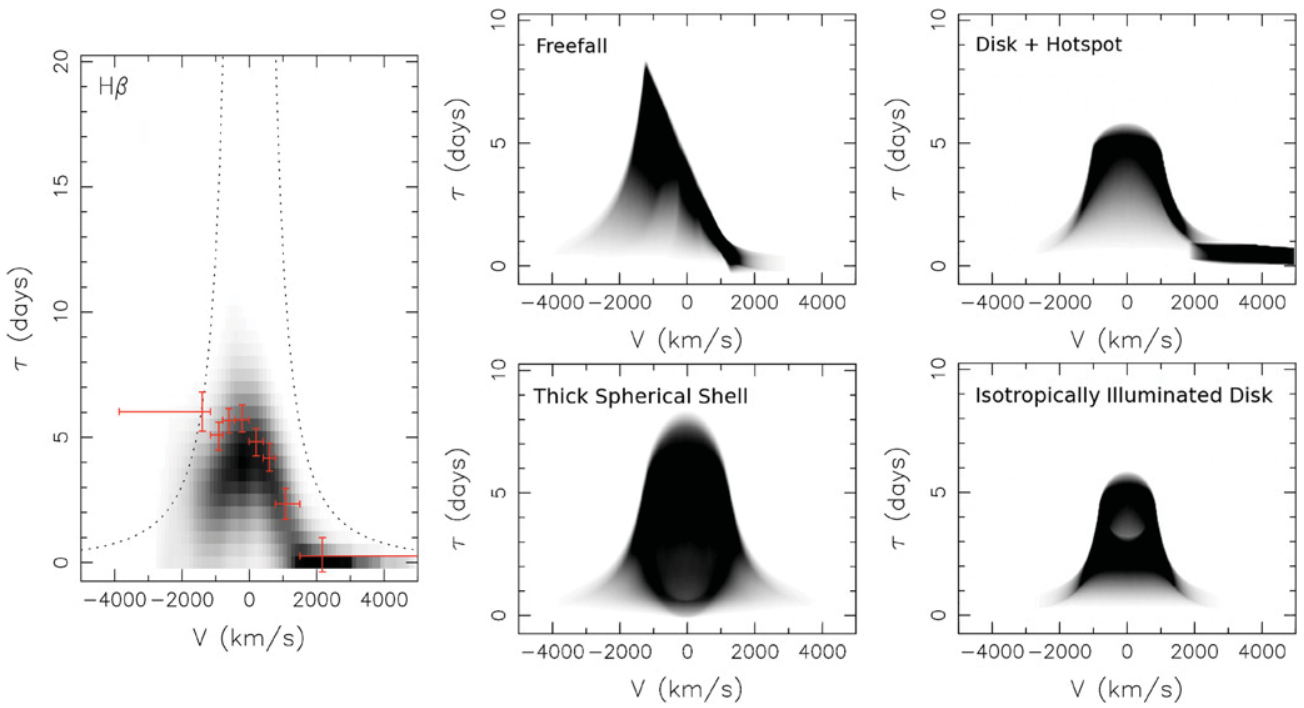

Figure 5. Velocity-delay map for $\mathrm{H} \beta$ compared to simple models for $M_{\mathrm{BH}}=7 \times 10^{6} M_{\odot}$ : (top) a free-falling BLR with inward emission anisotropy (left) and a partially illuminated disk with a hot spot at small radii on the receding side (right); (bottom) a biconically illuminated thick shell with circular orbits in a Keplerian potential and inward emission anisotropy (left) and a fully illuminated disk with inward emission anisotropy (right). The top models more accurately reproduce the features seen in the $\mathrm{H} \beta$ velocity-delay map than do the bottom models. All models have been smoothed to match the lower resolution of the recovered velocity-delay maps. The dotted lines in the $\mathrm{H} \beta$ panel again show the virial envelope and the red data points show the average lag measurement per velocity bin from Bentz et al. (2009).

(A color version of this figure is available in the online journal.)

of an inward radiation anisotropy (as displayed in Figure 5 with $F_{\text {in }} / F_{\text {total }}=0.8$ ) and/or by reducing the response of the inner gas. Models with radially outflowing gas have the opposite asymmetry and are thus largely ruled out.

For an inclined circular orbit, the velocity-delay structure is an ellipse with $\tau=(1+\sin i \cos \theta) R / c$ and $v=V \sin i \sin \theta$. The basic virial envelope, with a wider velocity range at smaller delays, can be reproduced by distributions of circular Keplerian orbits ranging from isotropically distributed angular momentum to inclined disks (e.g., the thick spherical shell and isotropically illuminated disk models in Figure 5). For such models, the Balmer response at small delay in the line core can be reduced by (1) anisotropic ionizing radiation directed toward the far side, (2) anisotropic inwardly directed line response, and/or (3) excluding edge-on orbits to form an inclined disk-like geometry. The enhanced prompt response in the red wing is hard to reproduce with a symmetric distribution of circular orbits. If the orbits circulate in the same direction, however, then the required asymmetry can be introduced by azimuthal structure-enhancing response (a "hot spot") on the receding side of the inner disk. While this is somewhat ad hoc, it might plausibly arise in the context of a warped-disk geometry, the warp exposing gas to ionizing radiation at small radius while shielding it at larger radius. The Disk + Hot spot model displayed in Figure 5 is inclined $\left(i=20^{\circ}\right)$ with $R_{\text {in }}=0.1$ lt-days and $R_{\text {out }}=4$ lt-days. No emission originates from the near side of the disk due to its warped shape, and excess emission arises on the receding portion of the inner disk at $0.1-0.3$ lt-days.

Both the Infall model and Disk + Hot spot model qualitatively reproduce the observed features of the velocity-delay maps, and more work is necessary to determine which family of models is preferred. In the meantime, we note that warped disks are seen in many astrophysical environments and have previously been invoked to explain various AGN phenomena (e.g., flux variations in the double-peaked broad emission from NGC 1097, Storchi-Bergmann et al. 1997; Fe emission-line profiles in the X-rays, Hartnoll \& Blackman 2000; the misalignment of radio jets and nuclear disks, Schmitt et al. 2002; and a self-contained apparatus for AGN unification, e.g., Nayakshin 2005). We consider here the plausibility of irradiation-induced warping (Pringle 1996), in which an optically thick, geometrically thin disk irradiated by a central source is unstable to warping from radiation pressure. The instability criterion is

$$
\frac{R}{R_{G}} \geqslant 8 \pi^{2} \eta^{2} \epsilon^{-1},
$$

where $R_{G}=G M_{\mathrm{BH}} / c^{2}, \eta$ is the ratio of the viscosities in the plane of and perpendicular to the disk (assumed here to be equal, i.e., $\eta=1$ ), and $\epsilon$ is the accretion efficiency. Assuming $\epsilon \approx 0.1$, a disk in the BLR of Arp 151 could become unstable on length scales $R / R_{G} \geqslant 800 \approx 0.3$ lt-day, inside the scale of the optical BLR. For typical estimates of BLR parameters (following Storchi-Bergmann et al. 1997), the precession timescale would likely be decades to centuries.

\section{SUMMARY}

We have recovered velocity-delay maps for six optical hydrogen and helium recombination lines in the LAMP spectra of Arp 151 using the maximum-entropy method. These are the most detailed velocity-delay maps constructed to date. The individual Balmer lines have similar velocity-delay structure, and the maps show responses with delays increasing from $\mathrm{H} \gamma$ to $\mathrm{H} \beta$ to $\mathrm{H} \alpha$. This can be interpreted as radial stratification through the Balmer series and is expected from optical-depth effects. All three lines show prompt response in their red wings, contrary to what has been seen in NGC 5548. The helium-line response is mostly unresolved in time.

The features seen in the Balmer-line velocity-delay maps can be reproduced qualitatively with either of two simple ad hoc models: a free-falling BLR or a partially illuminated thin disk with a localized excess of emission at small radii that could be interpreted as a warped disk. Warped disks are known to occur in many environments and on many astronomical scales, and it is 
plausible that the BLR in AGNs is another manifestation of this common configuration, as has been previously suggested in the literature. It appears to be impossible for a simple disk model or a thick spherical shell composed of randomly inclined circular orbits to accurately reproduce the features seen in the velocitydelay maps, including the strong emission at short lag times in the red wings of the Balmer lines and the lack of emission at short lag times in the line cores. Radial outflows are also largely ruled out by the observed asymmetric response.

The simple models and plausibility arguments presented here are the first steps in interpreting the velocity-delay maps for Arp 151. We are currently investigating other LAMP targets and expect to recover velocity-delay maps for a few additional objects, which may lead to insights into BLR differences across the Seyfert 1 population. Future monitoring programs will benefit from even higher temporal sampling and the avoidance of any gaps in the driving continuum light curve, allowing the driving light curve to provide stronger constraints and leading to more detailed velocity-delay maps of the emissionline responses. More sophisticated modeling will also help with interpreting the velocity-delay maps and determining which families of BLR models can be ruled out, allowing additional insights into the complexities of AGN BLRs.

We are grateful to Brad Peterson, Kelly Denney, and Kirk Korista for helpful conversations. M.C.B. thanks the University of St. Andrews for their hospitality and the STFC Rolling Grant to St. Andrews for supporting a visit that enabled the beginnings of this investigation. M.C.B. gratefully acknowledges support provided by NASA through Hubble Fellowship grant HF-51251 awarded by the Space Telescope Science Institute, which is operated by the Association of Universities for Research in Astronomy, Inc., for NASA, under contract NAS 5-26555. LAMP was supported by NSF grants AST-0548198 (UC Irvine), AST-0607485, AST-0908886 (UC Berkeley), AST-0642621 (UC Santa Barbara), and AST-0507450 (UC Riverside).

\section{REFERENCES}

Antonucci, R. R. J., \& Cohen, R. D. 1983, ApJ, 271, 564

Bentz, M. C., et al. 2008, ApJ, 689, L21

Bentz, M. C., et al. 2009, ApJ, 705, 199

Bentz, M. C., et al. 2010, ApJ, 716, 993

Blandford, R. D., \& McKee, C. F. 1982, ApJ, 255, 419

Bottorff, M., Korista, K. T., Shlosman, I., \& Blandford, R. D. 1997, ApJ, 479, 200

Chiang, J., \& Murray, N. 1996, ApJ, 466, 704

Crenshaw, D. M., \& Blackwell, J. H., Jr. 1990, ApJ, 358, L37

Denney, K. D., et al. 2010, ApJ, in press (arXiv:1006.4160)

Denney, K. D., et al. 2009, ApJ, 704, L80

Done, C., \& Krolik, J. H. 1996, ApJ, 463, 144

Gaskell, C. M. 1988, ApJ, 325, 114

Hartnoll, S. A., \& Blackman, E. G. 2000, MNRAS, 317, 880

Horne, K. 1994, in ASP Conf. Ser. 69, Reverberation Mapping of the Broad-line Region in Active Galactic Nuclei, ed. P. M. Gondhalekar, K. Horne, \& B. M. Peterson (San Francisco, CA: ASP), 23

Horne, K., Peterson, B. M., Collier, S. J., \& Netzer, H. 2004, PASP, 116 , 465

Horne, K., Welsh, W. F., \& Peterson, B. M. 1991, ApJ, 367, L5

Kaspi, S., Smith, P. S., Netzer, H., Maoz, D., Jannuzi, B. T., \& Giveon, U. 2000, ApJ, 533, 631

Koratkar, A. P., \& Gaskell, C. M. 1989, ApJ, 345, 637

Korista, K. T., \& Goad, M. R. 2004, ApJ, 606, 749 (erratum 627, 577 [2005])

Krolik, J. H., Horne, K., Kallman, T. R., Malkan, M. A., Edelson, R. A., \& Kriss, G. A. 1991, ApJ, 371, 54

Landolt, A. U. 1992, AJ, 104, 340

Matthews, T. A., \& Sandage, A. R. 1963, ApJ, 138, 30

Nayakshin, S. 2005, MNRAS, 359, 545

Peterson, B. M. 1993, PASP, 105, 247

Peterson, B. M., Wagner, R. M., Crenshaw, D. M., Meyers, K. A., Byard, P. L., Foltz, C. B., \& Miller, H. R. 1983, AJ, 88, 926

Peterson, B. M., et al. 2004, ApJ, 613, 682

Pringle, J. E. 1996, MNRAS, 281, 357

Schmitt, H. R., Pringle, J. E., Clarke, C. J., \& Kinney, A. L. 2002, ApJ, 575, 150

Smith, H. J., \& Hoffleit, D. 1963, Nature, 198, 650

Storchi-Bergmann, T., Eracleous, M., Ruiz, M. T., Livio, M., Wilson, A. S., \& Filippenko, A. V. 1997, ApJ, 489, 87

Ulrich, M.-H., \& Horne, K. 1996, MNRAS, 283, 748

Walsh, J. L., et al. 2009, ApJS, 185, 156

Wanders, I., et al. 1995, ApJ, 453, L87 